\title{
Important Databases Related to Genomes
}

\begin{abstract}
Genome databases are the locations, which permits storing, sharing, retrieving and comparison of the information related to the genomes of various individuals and organisms. Traditionally the databases were confined to the updated information of certain vital model organisms. Rapid development of technology and high speed internet facilities have created an explosion of databases resulting in the development of specific databases of almost all model organisms and a group of organisms with a common specificity. In the present chapter, details of the databases related to the genomes of viruses, archaea, bacteria, cell organelle, invertebrates, vertebrates, plants, and human beings are provided in a table format which provides an instant information, about different databases and their URLs.
\end{abstract}

\subsection{Virus Databases}

\begin{tabular}{l|l|l}
\hline Database & Description & URL \\
\hline CoVDB & Coronavirus genes and genomes & $\begin{array}{l}\text { http://covdb.microbiology.hku. } \\
\text { hk/ }\end{array}$ \\
\hline DPV web & $\begin{array}{l}\text { Central source of information about } \\
\text { viruses, viroids and satellites of plants, } \\
\text { fungi, and protozoa }\end{array}$ & http://www.dpvweb.net/ \\
\hline euHCVdb & European hepatitis C virus database & http://euhcvdb.ibcp.fr/ \\
\hline HCV database & Hepatitis C virus database & http://hcv.lanl.gov/ \\
\hline HERVd & Human endogenous retrovirus database & http://herv.img.cas.cz/ \\
\hline HFV database & $\begin{array}{l}\text { Hemorrhagic fever virus sequence } \\
\text { database }\end{array}$ & http://hfv.lanl.gov/ \\
\hline $\begin{array}{l}\text { HIV drug } \\
\text { resistance DB }\end{array}$ & $\begin{array}{l}\text { Compilation of mutations in HIV genes } \\
\text { that confer resistance to anti-HIV drugs }\end{array}$ & $\begin{array}{l}\text { http://www.hiv.lanl.gov/content/ } \\
\text { sequence/RESDB/ }\end{array}$ \\
\hline $\begin{array}{l}\text { Influenza } \\
\text { research DB }\end{array}$ & $\begin{array}{l}\text { Influenza virus } \\
\text { http://www.fludb.org }\end{array}$ \\
\hline
\end{tabular}

(continued) 


\begin{tabular}{l|l|l}
\hline $\begin{array}{l}\text { NCBI viral } \\
\text { genomes }\end{array}$ & Viral genomes resource & $\begin{array}{l}\text { http://www.ncbi.nlm.nih.gov/ } \\
\text { genomes/VIRUSES/viruses.html }\end{array}$ \\
\hline $\begin{array}{l}\text { Papillomavirus } \\
\text { episteme }\end{array}$ & $\begin{array}{l}\text { A database of Papillomaviridae family } \\
\text { of viruses }\end{array}$ & http://PaVE.niaid.nih.gov \\
\hline phiSITE & Gene regulation in bacteriophages & http://www.phisite.org \\
\hline Poxvirus.org & $\begin{array}{l}\text { Poxvirus genomic sequences and gene } \\
\text { annotation }\end{array}$ & http://www.poxvirus.org/ \\
\hline VBRC & Viral bioinformatics resource center & http://www.biovirus.org/ \\
\hline ViTa & $\begin{array}{l}\text { microRNAs targets of the influenza } \\
\text { virus }\end{array}$ & http://vita.mbc.nctu.edu.tw/ \\
\hline ViralZone & $\begin{array}{l}\text { Molecular and epidemiological } \\
\text { information on viral genera and families }\end{array}$ & http://viralzone.expasy.org/ \\
\hline ViralGenomes & Viral sequence data resource by NCBI & $\begin{array}{l}\text { https://www.ncbi.nlm.nih.gov/ } \\
\text { genome/viruses/ }\end{array}$ \\
\hline
\end{tabular}

\subsection{Archaeal Databases}

\begin{tabular}{|c|c|c|}
\hline Database & Description & URL \\
\hline Ensembl & A browser for archaeal genomes & $\begin{array}{l}\text { https://bacteria.ensembl.org/ } \\
\text { index.html }\end{array}$ \\
\hline EZ cloud & EZ cloud for archaea & $\begin{array}{l}\text { http://www.ezbiocloud.net/ } \\
\text { ezgenome }\end{array}$ \\
\hline IMG & $\begin{array}{l}\text { Integrated microbial genomes and } \\
\text { microbiomes }\end{array}$ & https://img.jgi.doe.gov/ \\
\hline $\begin{array}{l}\text { MicrobesOnline } \\
\text { resource }\end{array}$ & $\begin{array}{l}\text { A collection of } 3707 \text { microbial } \\
\text { genomes }\end{array}$ & http://www.microbesonline.org/ \\
\hline BacDive & $\begin{array}{l}\text { The bacterial diversity metadatabase } \\
\text { contains detailed information on more } \\
\text { than } 20,000 \text { strains of bacteria }\end{array}$ & https://bacdive.dsmz.de/ \\
\hline Wikipedia & List of sequenced archaeal genomes & $\begin{array}{l}\text { https://en.wikipedia.org/wiki/ } \\
\text { List_of_sequenced_archaeal_ } \\
\text { genomes }\end{array}$ \\
\hline $\begin{array}{l}\text { UCSC archaeal } \\
\text { genome browser }\end{array}$ & $\begin{array}{l}\text { Integrated genome browser for } \\
\text { archaeal genomes }\end{array}$ & http://archaea.ucsc.edu/ \\
\hline
\end{tabular}

\subsection{Bacterial Databases}

\begin{tabular}{l|l|l}
\hline Database & Description & URL \\
\hline Ensembl bacteria & $\begin{array}{l}\text { A browser for bacterial and archaeal } \\
\text { genomes }\end{array}$ & $\begin{array}{l}\text { https://bacteria.ensembl. } \\
\text { org/index.html }\end{array}$ \\
\hline MBGD & $\begin{array}{l}\text { Database for comparative analysis of } \\
\text { completely sequenced microbial } \\
\text { genomes }\end{array}$ & http://mbgd.genome.ad.jp/ \\
\hline $\begin{array}{l}\text { Human microbiome } \\
\text { project }\end{array}$ & $\begin{array}{l}\text { Characterisation of microbes in healthy } \\
\text { humans }\end{array}$ & https://hmpdacc.org/ \\
\hline
\end{tabular}




\begin{tabular}{|c|c|c|}
\hline GOLD & Genomes online database & https://gold.jgi.doe.gov/ \\
\hline EZ cloud & EZ cloud for bacteria & $\begin{array}{l}\text { http://www.ezbiocloud.net/ } \\
\text { ezgenome }\end{array}$ \\
\hline IMG & $\begin{array}{l}\text { Integrated microbial genomes and } \\
\text { microbiomes }\end{array}$ & https://img.jgi.doe.gov/ \\
\hline $\begin{array}{l}\text { MicrobesOnline } \\
\text { resource }\end{array}$ & $\begin{array}{l}\text { A collection of } 3707 \text { mmicrobial } \\
\text { genomes }\end{array}$ & $\begin{array}{l}\text { http://www. } \\
\text { microbesonline.org/ }\end{array}$ \\
\hline BacDive & $\begin{array}{l}\text { The bacterial diversity metadatabase } \\
\text { contains detailed information on more } \\
\text { than } 20,000 \text { strains of bacteria }\end{array}$ & https://bacdive.dsmz.de/ \\
\hline BIGSdb & $\begin{array}{l}\text { Bacterial isolate genome sequence } \\
\text { database }\end{array}$ & $\begin{array}{l}\text { https://bigsdb.readthedocs. } \\
\text { io/en/latest/ }\end{array}$ \\
\hline metaMicrobesOnline & $\begin{array}{l}\text { Website for browsing and comparing } \\
\text { the genomes of } 3527 \text { microbial } \\
\text { genomes }\end{array}$ & $\begin{array}{l}\text { http://meta.microbesonline. } \\
\text { org/ }\end{array}$ \\
\hline MetaRef & $\begin{array}{l}\text { A pan-genomic database for } \\
\text { comparative and community microbial } \\
\text { genomics }\end{array}$ & $\begin{array}{l}\text { https://omictools.com/ } \\
\text { metaref-tool }\end{array}$ \\
\hline MetaBioME database & $\begin{array}{l}\text { MetaBioME is a web resource to find } \\
\text { novel homologs for known } \\
\text { commercially useful enzymes (CUEs) } \\
\text { in metagenomic datasets and completed } \\
\text { bacterial genomes }\end{array}$ & $\begin{array}{l}\text { https://metasystems.riken. } \\
\text { jp/metabiome/ }\end{array}$ \\
\hline PATRIC & $\begin{array}{l}\text { Pathosystems resource integration } \\
\text { center }\end{array}$ & https://www.patricbrc.org/ \\
\hline TBDB & $\begin{array}{l}\text { Tuberculosis database integrates } \\
\text { genomic sequences and data for } \\
\text { Mycobacterium species relevant to drug } \\
\text { discovery, vaccines, and biomarkers }\end{array}$ & http://www.tbdb.org/ \\
\hline EuPathDB & $\begin{array}{l}\text { Eukaryotic pathogen database resources } \\
\text { is a collection of individual databases, } \\
\text { each focusing on specific pathogens, } \\
\text { accessible through a common portal }\end{array}$ & https://eupathdb.org/ \\
\hline coliBase & $\begin{array}{l}\text { A database for E. coli, Salmonella and } \\
\text { Shigella }\end{array}$ & $\begin{array}{l}\text { http://xbase.warwick.ac.uk/ } \\
\text { colibase/ }\end{array}$ \\
\hline DEG & $\begin{array}{l}\text { Database of essential genes from } \\
\text { bacteria and yeast }\end{array}$ & $\begin{array}{l}\text { http://www.essentialgene. } \\
\text { org }\end{array}$ \\
\hline $\begin{array}{l}\text { Essential genes in } E \text {. } \\
\text { coli }\end{array}$ & $\begin{array}{l}\text { First results of an E. coli gene deletion } \\
\text { project }\end{array}$ & $\begin{array}{l}\text { http://www.genome.wisc. } \\
\text { edu/resources/essential.htm }\end{array}$ \\
\hline GenoBase & $\begin{array}{l}\text { Escherichia coli K-12 in-frame, single- } \\
\text { gene knockout mutants }\end{array}$ & http://ecoli.naist.jp/ \\
\hline HGT-DB & $\begin{array}{l}\text { Horizantal gene transfer database is a } \\
\text { genomics database that includes } \mathrm{G}+\mathrm{C} \\
\text { content, codon and amino-acid usage, } \\
\text { as well as information on which genes } \\
\text { deviate in these parameters for } \\
\text { prokaryotic complete genomes }\end{array}$ & $\begin{array}{l}\text { http://genomes.urv.es/ } \\
\text { HGT-DB/ }\end{array}$ \\
\hline $\begin{array}{l}\text { Pseudomonas genome } \\
\text { database }\end{array}$ & $\begin{array}{l}\text { Consists of information about } \\
\text { Pseudomonas genome }\end{array}$ & $\begin{array}{l}\text { http://www.pseudomonas. } \\
\text { com// }\end{array}$ \\
\hline PAIDB & Pathogeneticity islands database & $\begin{array}{l}\text { http://www.paidb.re.kr/ } \\
\text { about_paidb.php }\end{array}$ \\
\hline
\end{tabular}




\subsection{Cell Organelle Databases}

\begin{tabular}{l|l|l}
\hline Database & Description & URL \\
\hline $\begin{array}{l}\text { Chloroplast } \\
\text { genome database }\end{array}$ & Chloroplast genome & http://chloroplast.cbio.psu.edu/ \\
\hline OrganelleDB & $\begin{array}{l}\text { A web-accessible database cataloging } \\
\text { proteins localized to a known organelle }\end{array}$ & http://organelledb.lsi.umich.edu/ \\
\hline $\begin{array}{l}\text { Organelle } \\
\text { genomes }\end{array}$ & Organelle genome resource at NCBI & www.ncbi.nlm.nih.gov/genomes/ \\
ORGANELLES/organelles.html \\
\hline $\begin{array}{l}\text { Plant organelles } \\
\text { database }\end{array}$ & $\begin{array}{l}\text { Images of plant organelles and } \\
\text { protocols for plant organelle research }\end{array}$ & $\begin{array}{l}\text { http://podb.nibb.ac.jp/ } \\
\text { Organellome/ }\end{array}$ \\
\hline PLProt & $\begin{array}{l}\text { Arabidopsis thaliana chloroplast } \\
\text { protein database }\end{array}$ & http://www.plprot.ethz.ch/ \\
\hline $\begin{array}{l}\text { Plastid-LCG } \\
\text { base }\end{array}$ & $\begin{array}{l}\text { Plastid lineage-based conserved gene- } \\
\text { pair database }\end{array}$ & http://lcgbase.big.ac.cn/plastid- \\
LCGbase/
\end{tabular}

\subsection{In-Vertebrate Genome Databases}

\begin{tabular}{l|l|l}
\hline Database & Description & URL \\
\hline AphidBase & $\begin{array}{l}\text { Genome database of pea aphid } \\
\text { (Acyrthosiphon pisum) }\end{array}$ & http://www.aphidbase.com/ \\
\hline AppaDB & $\begin{array}{l}\text { Database of nematode Pristionchus } \\
\text { pacificus }\end{array}$ & $\begin{array}{l}\text { http://appadb.eb.tuebingen.mpg. } \\
\text { de }\end{array}$ \\
\hline BeetleBase & $\begin{array}{l}\text { Database of the beetle Tribolium } \\
\text { castaneum }\end{array}$ & http://www.beetlebase.org \\
\hline CeNDR & C. elegans natural diversity resource & http://www.elegansvariation.org \\
\hline
\end{tabular}




\begin{tabular}{|c|c|c|}
\hline Comparasite & $\begin{array}{l}\text { Database for comparative studies of } \\
\text { transcriptomes of parasites }\end{array}$ & http://comparasite.hgc.jp/ \\
\hline CuticleDB & Structural proteins of arthropod cuticle & $\begin{array}{l}\text { http://bioinformatics.biol.uoa.gr/ } \\
\text { cuticleDB/ }\end{array}$ \\
\hline FlyBrain & Database of drosophila nervous system & $\begin{array}{l}\text { http://flybrain.neurobio.arizona. } \\
\text { edu/ }\end{array}$ \\
\hline Helminth.net & Parasitic nematode sequencing project & http://www.helminth.net \\
\hline InsectBase & Insect genomes and transcriptomes & http://www.insect-genome.com/ \\
\hline $\begin{array}{l}\text { Hymenoptera } \\
\text { genome }\end{array}$ & $\begin{array}{l}\text { Genome sequences and annotation for } \\
\text { honey bee and the parasitoid wasp } \\
\text { Nasonia vitripennis }\end{array}$ & http://HymenopteraGenome.org \\
\hline InsectBase & Insect genomes and transcriptomes & http://www.insect-genome.com/ \\
\hline SilksatDB & $\begin{array}{l}\text { Comprehensive relational database of } \\
\text { silkworm microsatellite }\end{array}$ & $\begin{array}{l}\text { http://www.cdfd.org.in/ } \\
\text { SILKSAT/index.php }\end{array}$ \\
\hline SpodoBase & $\begin{array}{l}\text { Genomics of the butterfly Spodoptera } \\
\text { frugiperda }\end{array}$ & $\begin{array}{l}\text { http://bioweb.ensam.inra.fr/ } \\
\text { spodobase/ }\end{array}$ \\
\hline VectorBase & Invertebrate vectors of human pathogens & http://www.vectorbase.org \\
\hline RNAiDB & $\begin{array}{l}\text { RNAi phenotypic analysis of C. elegans } \\
\text { genes }\end{array}$ & http://www.rnai.org/ \\
\hline NEMBASE & $\begin{array}{l}\text { Nematode sequence and functional data } \\
\text { database }\end{array}$ & http://www.nematodes.org/ \\
\hline PLANMINE & $\begin{array}{l}\text { Platform for archiving and mining } \\
\text { planarian biodiversity }\end{array}$ & http://planmine.mpi-cbg.de/ \\
\hline WORMBASE & Database of Caenorhabditis elegans & http://www.wormbase.org \\
\hline WorfDB & $\begin{array}{l}\text { Worm ORF database which contains } \\
\text { predicted proteins from C. elegans }\end{array}$ & http://worfdb.dfci.harvard.edu/ \\
\hline
\end{tabular}

\subsection{Vertebrate Genome Databases}

\begin{tabular}{l|l|l}
\hline Database & Description & URL \\
\hline FANTOM & $\begin{array}{l}\text { Functional annotation of mouse full-length } \\
\text { cDNA clones }\end{array}$ & http://fantom.gsc.riken.jp/ \\
\hline GWIPS-viz & Genomes of human beings and vertebrates & http://gwips.ucc.ie \\
\hline Xenbase & Human and Vertebrate Genomes & http://www.xenbase.org \\
\hline $\begin{array}{l}\text { Mammalian } \\
\text { gene } \\
\text { collection }\end{array}$ & $\begin{array}{l}\text { Identify and sequence cDNA clones of FL } \\
\text { or ORF for human, mouse and rat genes }\end{array}$ & http://mgc.nci.nih.gov/ \\
\hline PlasmID & $\begin{array}{l}\text { A repository for collection and distribution } \\
\text { of plasmid clones for human and vertebrate } \\
\text { genomes }\end{array}$ & http://plasmid.hms.harvard.edu/ \\
\hline $\begin{array}{l}\text { Mouse } \\
\text { genome } \\
\text { database }\end{array}$ & $\begin{array}{l}\text { Mouse genome database for integrated data } \\
\text { on the genetics, genomics and biology of } \\
\text { the laboratory mouse }\end{array}$ & http://www.informatics.jax.org \\
\hline
\end{tabular}




\subsection{Human Genome Databases}

\begin{tabular}{|c|c|c|}
\hline Database & Description & URL \\
\hline 3DSNP & $\begin{array}{l}\text { Database for comprehensively annotating } \\
\text { the regulatory function of human } \\
\text { noncoding SNPs by exploring their 3D } \\
\text { interactions with genes and genetically } \\
\text { associated SNPs mediated by chromatin } \\
\text { loops }\end{array}$ & http://biotech.bmi.ac.cn/3dsnp/ \\
\hline $\begin{array}{l}\text { ENCODE at } \\
\text { UCSC }\end{array}$ & $\begin{array}{l}\text { Encyclopedia of DNA elements an } \\
\text { international a consortium of } \\
\text { investigators funded to analyze the } \\
\text { human genome with the goal of } \\
\text { producing a comprehensive catalog of } \\
\text { functional elements }\end{array}$ & $\begin{array}{l}\text { http://genome.ucsc.edu/ } \\
\text { ENCODE }\end{array}$ \\
\hline GeneAnnot & $\begin{array}{l}\text { Revises and improves the annotation of } \\
\text { affymetrix probe sets }\end{array}$ & $\begin{array}{l}\text { http://genecards.weizmann.ac.il/ } \\
\text { geneannot/ }\end{array}$ \\
\hline $\begin{array}{l}\text { Locus } \\
\text { reference } \\
\text { genome } \\
\text { sequences }\end{array}$ & $\begin{array}{l}\text { Each sequence is a stable genomic DNA } \\
\text { sequence for a region of the human } \\
\text { genome }\end{array}$ & http://www.lrg-sequence.org \\
\hline $\begin{array}{l}\text { QTL match } \\
\text { maker }\end{array}$ & $\begin{array}{l}\text { Database which integrates QTLs of } \\
\text { human mouse and rat genomes to } \\
\text { annotate their functional genomes }\end{array}$ & $\begin{array}{l}\text { http://pmrc.med.mssm.edu:9090/ } \\
\text { QTL/jsp/qtlhome.jsp }\end{array}$ \\
\hline TIARA & $\begin{array}{l}\text { Total integrated archive of short-read and } \\
\text { array (TIARA) accumulates raw-level } \\
\text { personal genomic data from whole } \\
\text { genome next-generation sequencing } \\
\text { (NGS) and comparative genomic } \\
\text { hybridization (CGH) arrays }\end{array}$ & http://tiara.gmi.ac.kr \\
\hline TRBASE & $\begin{array}{l}\text { Database that relates tandem repeats to } \\
\text { the gene locations and disease genes in } \\
\text { the human genome }\end{array}$ & http://trbase.ex.ac.uk/ \\
\hline UniSTS & $\begin{array}{l}\text { A comprehensive database of sequence } \\
\text { tagged sites (STSs), each of which is } \\
\text { defined by a distinct pair of primer } \\
\text { sequences designed for use in a PCR } \\
\text { reaction }\end{array}$ & $\begin{array}{l}\text { http://www.ncbi.nlm.nih.gov/ } \\
\text { probe }\end{array}$ \\
\hline $\mathrm{X}: \mathrm{MaP}$ & $\begin{array}{l}\text { Annotation and visualization of genome } \\
\text { structure for Affymetrix exon array } \\
\text { analysis }\end{array}$ & http://annmap.picr.man.ac.uk/ \\
\hline EVOLA & $\begin{array}{l}\text { A database of human genes and their } \\
\text { vertebrate orthologs }\end{array}$ & http://jbirc.jbic.or.jp/hinv/evola/ \\
\hline H-InvDB & $\begin{array}{l}\text { HumanInvitational database is an } \\
\text { integrated database of human genes and } \\
\text { transcripts }\end{array}$ & http://www.h-invitational.jp/ \\
\hline HGPD & $\begin{array}{l}\text { Human gene and Protein database is a } \\
\text { unique database that stores information } \\
\text { from human gateway entry clones and } \\
\text { in vitro expression data related to human } \\
\text { proteins }\end{array}$ & $\begin{array}{l}\text { http://hupex.hgpd.jp/entrance/ } \\
\text { index.html }\end{array}$ \\
\hline
\end{tabular}




\begin{tabular}{l|l|l}
\hline HRPD & $\begin{array}{l}\text { Human protein reference database that } \\
\text { stores the data related to domain } \\
\text { architecture, post-translational } \\
\text { modifications, interaction networks and } \\
\text { disease }\end{array}$ & http://www.hprd.org/ \\
\hline LIFEdb & $\begin{array}{l}\text { A database on the localisation, } \\
\text { interaction, functional assays and } \\
\text { expression of human proteins }\end{array}$ & $\begin{array}{l}\text { http://www.dkfz.de/gpcf/lifedb. } \\
\text { php }\end{array}$ \\
\hline NetAffx & $\begin{array}{l}\text { A database of public affymetrix probesets } \\
\text { and annotations }\end{array}$ & http://www.affymetrix.com \\
\hline $\begin{array}{l}\text { ORFDB/ } \\
\text { ORFeome }\end{array}$ & $\begin{array}{l}\text { Invitrogen initiated program intended to } \\
\text { provide the open reading frames (ORFs) } \\
\text { of all human proteins }\end{array}$ & $\mathrm{http} / / /$ orf.invitrogen.com/ \\
\hline dbGaP & $\begin{array}{l}\text { Database of genotypes and phenotypes } \\
\text { MSY } \\
\text { breakpoint } \\
\text { mapper }\end{array}$ & $\begin{array}{l}\text { STSs on human Y chromosome } \\
\text { http://www.ncbi.nlm.nih.gov/gap }\end{array}$ \\
\hline Gene cards & $\begin{array}{l}\text { An automated, integrated database of } \\
\text { human genes, genomic maps, proteins } \\
\text { and diseases }\end{array}$ & $\mathrm{http} / / / \mathrm{www} \cdot$ genecards.org/ \\
\hline
\end{tabular}

\subsection{Plant Genome Databases}

\begin{tabular}{|c|c|c|}
\hline Database & Description & URL \\
\hline AgBase & $\begin{array}{l}\text { A curated, open-source, web-accessible } \\
\text { resource for functional analysis of } \\
\text { agricultural plant and animal gene } \\
\text { products }\end{array}$ & http://www.agbase.msstate.edu/ \\
\hline autoSNPdb & Plant SNP discovery database & $\begin{array}{l}\text { http://autosnpdb. } \\
\text { appliedbioinformatics.com.au/ }\end{array}$ \\
\hline BarleyBase & $\begin{array}{l}\text { Database for barley microarrays holds the } \\
\text { data for over } 1000 \text { hybridizations from } \\
\text { the affymetrix Barley } 1 \text { GeneChip and } \\
\text { genome arrays }\end{array}$ & http://www.barleybase.org/ \\
\hline $\begin{array}{l}\text { Cereal small } \\
\text { RNA database }\end{array}$ & $\begin{array}{l}\text { An integrated resource for small RNAs } \\
\text { such as miRNAs, siRNAs, ta-siRNAs } \\
\text { expressed in rice and maize }\end{array}$ & $\begin{array}{l}\text { http://sundarlab.ucdavis.edu/ } \\
\text { smrnas/ }\end{array}$ \\
\hline CR-EST & Crop EST database & $\begin{array}{l}\text { https://apex.ipk-gatersleben.de/ } \\
\text { apex/f?p=116:1 }\end{array}$ \\
\hline EURISCO & $\begin{array}{l}\text { European catalogue for plant genetic } \\
\text { resources }\end{array}$ & http://eurisco.ecpgr.org/ \\
\hline GABiPD & $\begin{array}{l}\text { Genome analysis of the plant biological } \\
\text { system }\end{array}$ & http://www.gabipd.org/ \\
\hline GoMapMan & Unified plant-specific gene ontology & http://www.gomapman.org \\
\hline GrainGenes & $\begin{array}{l}\text { Molecular and phenotypic information } \\
\text { on grain yielding plants such as wheat, } \\
\text { barley, rye, triticale, and oats }\end{array}$ & http://wheat.pw.usda.gov/ \\
\hline
\end{tabular}




\begin{tabular}{|c|c|c|}
\hline GreeNC & $\begin{array}{l}\text { Green non-coding database (GreeNC) is } \\
\text { a repository of long non-coding RNAs } \\
\text { annotated in } 37 \text { plant species and six } \\
\text { algae }\end{array}$ & $\begin{array}{l}\text { http://greenc.sciencedesigners. } \\
\text { com/wiki/Main_Page }\end{array}$ \\
\hline $\begin{array}{l}\text { MIPS plants } \\
\text { database }\end{array}$ & $\begin{array}{l}\text { Plant database resource for integrative } \\
\text { and comparative plant genome research }\end{array}$ & http://mips.gsf.de/proj/plant/jsf/ \\
\hline NIAS & $\begin{array}{l}\text { Databases for plant genetic resources and } \\
\text { plant disease information }\end{array}$ & $\begin{array}{l}\text { http://www.gene.affrc.go.jp/ } \\
\text { databases_en.php }\end{array}$ \\
\hline P-MITE & $\begin{array}{l}\text { Database of plant miniature inverted- } \\
\text { repeat transposable elements (MITEs) }\end{array}$ & $\begin{array}{l}\text { http://pmite.hzau.edu.cn/django/ } \\
\text { mite/ }\end{array}$ \\
\hline PGDD & A plant genome duplication database & $\begin{array}{l}\text { http://chibba.agtec.uga.edu/ } \\
\text { duplication/ }\end{array}$ \\
\hline Phytozome & A database for green plant genomics & http://www.phytozome.net/ \\
\hline PIECE & $\begin{array}{l}\text { Database of plant intron exon comparison } \\
\text { and evolution }\end{array}$ & https://wheat.pw.usda.gov/piece/ \\
\hline $\begin{array}{l}\text { Plant DNA } \\
\text { C-values } \\
\text { database }\end{array}$ & $\begin{array}{l}\text { A database that provides a one-stop, user- } \\
\text { friendly database where the plant genome } \\
\text { sizes can be readily accessed and } \\
\text { compared }\end{array}$ & $\begin{array}{l}\text { http://www.kew.org/genomesize/ } \\
\text { homepage.html }\end{array}$ \\
\hline $\begin{array}{l}\text { Plant ontology } \\
\text { database }\end{array}$ & $\begin{array}{l}\text { This database is a collection of plant } \\
\text { systematics, botany and genomics } \\
\text { involved in developing simple and } \\
\text { controlled vocabularies that accurately } \\
\text { reflect the biology of plant structures }\end{array}$ & http://www.plantontology.org/ \\
\hline $\begin{array}{l}\text { Plant } \\
\text { SnoRNA } \\
\text { database }\end{array}$ & $\begin{array}{l}\text { A database of snoRNA genes in plant } \\
\text { species }\end{array}$ & $\begin{array}{l}\text { http://bioinf.scri.sari.ac.uk/cgi- } \\
\text { bin/plant_snorna/home }\end{array}$ \\
\hline $\begin{array}{l}\text { Plant stress- } \\
\text { responsive } \\
\text { gene catalog }\end{array}$ & $\begin{array}{l}\text { Stress-responsive gene in various plant } \\
\text { species }\end{array}$ & http://dayhoff.generationcp.org/ \\
\hline Plant GDB & $\begin{array}{l}\text { A database of plant genomic sequences } \\
\text { and ESTs }\end{array}$ & http://www.plantgdb.org/ \\
\hline Plant PAN & $\begin{array}{l}\text { Plant promoter analysis navigator } \\
\text { database }\end{array}$ & $\begin{array}{l}\text { http://PlantPAN2.itps.ncku.edu. } \\
\text { tw }\end{array}$ \\
\hline PlantProm & A database of plant promoter sequences & http://www.epd.isb-sib.ch/ \\
\hline $\begin{array}{l}\text { PlantsP/ } \\
\text { PlantsT }\end{array}$ & $\begin{array}{l}\text { PlantsP and PlantsT are plant-specific } \\
\text { curated databases that combine sequence } \\
\text { derived information with experimental } \\
\text { functional genomics data }\end{array}$ & http://plantsp.sdsc.edu/ \\
\hline Plant tribes & $\begin{array}{l}\text { Families of protein-coding genes from } \\
\text { five sequenced plant species }\end{array}$ & $\begin{array}{l}\text { http://fgp.huck.psu.edu/tribedb/ } \\
\text { index.pl }\end{array}$ \\
\hline PlantTFDB & $\begin{array}{l}\text { Plant transcription factor database } \\
\text { provides a comprehensive, high-quality } \\
\text { resource of plant transcription factors, } \\
\text { regulatory elements and interactions } \\
\text { between them }\end{array}$ & http://planttfdb.cbi.pku.edu.cn \\
\hline PLAZA & $\begin{array}{l}\text { A plant-oriented online resource for } \\
\text { comparative, evolutionary and functional } \\
\text { genomics }\end{array}$ & $\begin{array}{l}\text { https://bioinformatics.psb.ugent. } \\
\text { be/plaza }\end{array}$ \\
\hline
\end{tabular}




\begin{tabular}{|c|c|c|}
\hline PMDBase & $\begin{array}{l}\text { A database of plant microsatellites and } \\
\text { marker development }\end{array}$ & $\begin{array}{l}\text { http://www.sesame-bioinfo.org/ } \\
\text { PMDBase }\end{array}$ \\
\hline $\begin{array}{l}\text { TIGR } \\
\text { database }\end{array}$ & Plant transcript assemblies database & http://plantta.tigr.org/ \\
\hline TROPGene & $\begin{array}{l}\text { A database comprising of genetic and } \\
\text { genomic information about tropical crops } \\
\text { such as banana, coconut, cotton, cocoa, } \\
\text { etc. }\end{array}$ & http://tropgenedb.cirad.fr/ \\
\hline ForestTree & A resource that centralizes large-scale & http://foresttree.org/ftdb \\
\hline DataBase & $\begin{array}{l}\text { EST sequencing results from several tree } \\
\text { species }\end{array}$ & \\
\hline LegumelIP & $\begin{array}{l}\text { Model legumes integrative database } \\
\text { platform }\end{array}$ & $\begin{array}{l}\text { http://plantgrn.noble.org/ } \\
\text { LegumeIP/ }\end{array}$ \\
\hline
\end{tabular}

\subsection{Genomes of Economically Important and Model Plants}

\begin{tabular}{l|l}
\hline Database & URL \\
\hline Medicago trunculata database & http://www.medicago.org/ \\
\hline Brassica genome & http://www.brassicagenome.net/ \\
\hline Coffee genome hub & http://coffee-genome.org/ \\
\hline CottonGen & http://www.cottongen.org \\
\hline $\begin{array}{l}\text { Maize genetics and genomics database } \\
\text { (MaizeGDB) }\end{array}$ & http://www.maizegdb.org/ \\
\hline The Arabidopsis information resource (TAIR) & https://www.arabidopsis.org/ \\
\hline Oryza sativa (ssp. japonica) genome & http://www.plantgdb.org/OsGDB/ \\
\hline The rice annotation project database & https://rapdb.dna.affrc.go.jp/ \\
\hline PoMaMo-potato maps and more & http://www.gabipd.org/projects/Pomamo/ \\
\hline Shanghai rapeseed database & http://rapeseed.plantsignal.cn/ \\
\hline Genome database for Rosaceae & http://www.rosaceae.org \\
\hline Solanaceae genomics network & http://solgenomics.net/ \\
\hline SoyBase & http://soybase.org/ \\
\hline SoyKB-soybean knowledge base & http://soykb.org \\
\hline TED—tomato functional genomics database & http://ted.bti.cornell.edu/ \\
\hline TIGR maize database & http://maize.tigr.org/ \\
\hline TomatEST DB & http://biosrv.cab.unina.it/tomatestdb/ \\
\hline TomatEST DB & http://biosrv.cab.unina.it/tomatestdb/ \\
\hline PANZEA & http://www.panzea.org/ \\
\hline Diatom EST database & http://www.biologie.ens.fr/diatomics/ \\
& EST/ \\
\hline
\end{tabular}

